In der Rubrik „Literatur kompakt" werden die wichtigsten Originalarbeiten aus der internationalen Fachliteratur referiert.

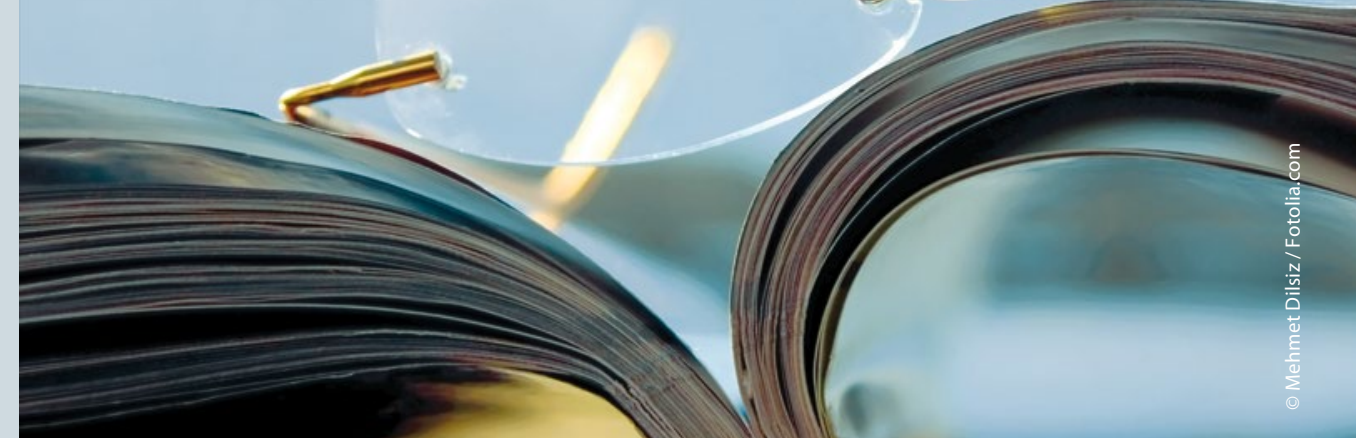

\section{Blasenkarzinom und Organerhalt: Manche Patienten profitieren von Chemoradiotherapie}

\author{
Die Kombination aus Chemotherapie und Bestrahlung ist bei bestimmten \\ Patienten mit organbegrenztem muskelinvasivem Blasenkrebs eine Strategie \\ zur organerhaltenden Versorgung.
}

aut McHaffie et al. existieren bisher - keine randomisierten Studien, in denen bei muskelinvasivem Blasenkarzinom die Wirksamkeit einer radikalen Zystektomie mit der einer cisplatinhaltigen Chemoradiotherapie zur Organerhaltung verglichen wurde. Deshalb analysierten die Wissenschaftler publizierte klinische Studien zum Nutzen organerhaltender Strategien. Im Fokus standen unter anderem sechs prospektive Studien der Radiation Therapy Oncology Group (RTOG) mit insgesamt 468 Patienten. Vor der Chemoradiotherapie wurde jeweils der Tumor transurethral entfernt. Bei Patienten ohne ein komplettes Ansprechen auf die Behandlung wurde die Blase komplett reseziert.

Das mediane Follow-up aller Patienten lag bei 4,3 Jahren. Es betrug 7,8 Jahre bei den Patienten, die zum Zeitpunkt der Auswertung noch lebten. Die krankheitsspezifische Überlebensrate lag nach fünf beziehungsweise zehn Jahren bei $71 \%$ beziehungsweise $61 \%$. Diese Raten seien ähnlich denen nach einer primären Zystektomie, so die Ärzte. Von den Patienten, die fünf Jahre nach der Therapie noch lebten - das waren 205 Studienteilnehmer -, hatten $80 \%$ eine funktionsfähige Harnblase. Die Studienautoren weisen darauf hin, dass diese Ergebnisse mit den Erfahrungen an einzelnen Kliniken (bspw. Universität Erlangen) übereinstimmen.
Weil die meisten Patienten, die für eine primäre Bestrahlung vorgesehen sind, älter sind und einen reduzierten Allgemeinzustand mit einer eingeschränkten Nierenfunktion haben, war man auf der Suche nach Chemotherapeutika zur Erhöhung der Strahlensensitivität, zumal gerade Patienten über 75 Jahre seltener eine Cisplatintherapie beendeten. Erste Erfolge mit 5-Fluorouracil (5-FU) plus Mitomycin C waren Anlass für die Studie Bladder Cancer 2001 (BC2001), in der die Wirksamkeit einer Bestrahlung mit der einer Bestrahlung

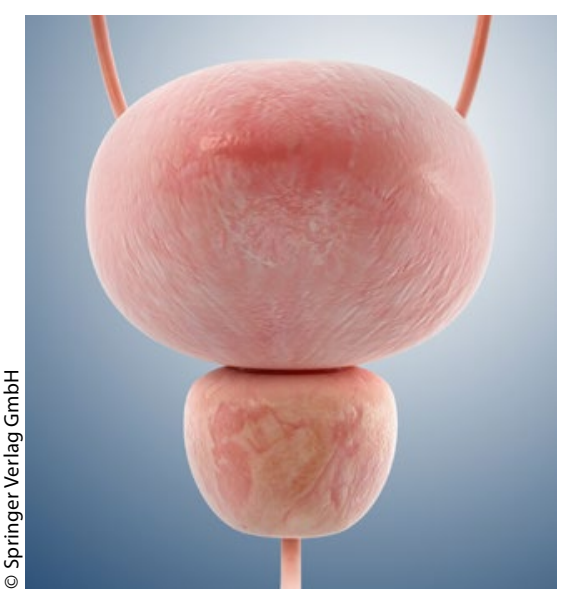

Für ein organerhaltendes Vorgehen bei Blasenkarzinomen sind nur bestimmte Patienten geeignet. in Kombination mit den beiden Präparaten geprüft wurde. An der britischen Studie nahmen 458 Patienten im medianen Alter von 72 Jahren teil. Durch die zusätzliche Chemotherapie wurde das lokoregionale krankheitsfreie Überleben signifikant verbessert (67\% versus 54 \%; Hazard Ratio: 0,68; 95\%-Konfidenzintervall zwischen 0,48 und 0,96; $\mathrm{p}=0,03$ ). Zudem war die Zystektomierate nach zwei Jahren deutlich niedriger $(11,4 \%$ vs. $16,8 \%)$. Bei der Gesamtüberlebensrate nach fünf Jahren war der Unterschied jedoch nicht signifikant (48\% vs. $35 \%)$.

Fazit: Die US-Ärzte erinnern daran, dass bei der Entscheidung für ein organerhaltendes Vorgehen die Patienten sorgfältig ausgewählt werden müssen. So hätten an den vorgestellten Studien nur Patienten mit muskelinvasiven, nodalnegativen T2bis T4a-Tumoren teilgenommen. Einschränkend plädieren sie dafür, Tumoren im Stadium T4a - also mit Invasion in Nachbarorgane wie die Prostata - als Kontraindikation für das organerhaltende Vorgehen zu betrachten, da Patienten mit solchen Tumoren in den Studien RTOG und BC2001 mit 3,9 \% unterrepräsentiert gewesen seien. Schließlich gehe aus den Studien auch hervor, dass eine Hydronephrose mit einer schlechten Prognose einhergehe.

In Deutschland wird die S3-Leitline zum Harnblasenkarzinom unter Federführung der Deutschen Gesellschaft für Urologie in Kürze veröffentlicht.

Peter Leiner

McHaffie DR et al. Chemoradiation for organ preservation in the treatment of muscle-invasive bladder cancer. Urol Oncol. 2016;online 20. April;doi:10.1016/j.urolonc.2016.03.011. 